\title{
Supersymmetric Majoron inflation
}

\section{Stephen F. King and Patrick Otto Ludl}

School of Physics and Astronomy, University of Southampton, Southampton, SO17 1BJ, U.K.

E-mail: king@soton.ac.uk, P.Ludl@soton.ac.uk

AbStract: We propose supersymmetric Majoron inflation in which the Majoron field $\Phi$ responsible for generating right-handed neutrino masses may also be suitable for giving low scale "hilltop" inflation, with a discrete lepton number $\mathbb{Z}_{N}$ spontaneously broken at the end of inflation, while avoiding the domain wall problem. In the framework of nonminimal supergravity, we show that a successful spectral index can result with small running together with small tensor modes. We show that a range of heaviest right-handed neutrino masses can be generated, $m_{N} \sim 10^{1}-10^{16} \mathrm{GeV}$, consistent with the constraints from reheating and domain walls.

Keywords: Cosmology of Theories beyond the SM, Neutrino Physics

ARXIV EPRINT: 1701.04794 


\section{Contents}

1 Introduction 1

2 The model with non-minimal Kähler potential 3

3 Majoron inflation $\quad 7$

$\begin{array}{ll}3.1 & \text { The domain wall problem }\end{array}$

$\begin{array}{ll}3.1 .1 & \text { The height of the barrier }\end{array}$

3.1.2 The reheat temperature 8

3.1.3 Creation of domain walls after inflation 9

$\begin{array}{ll}3.2 & \text { Inflation phenomenology } \\ 3.31\end{array}$

$\begin{array}{ll}3.3 & \text { Number of e-folds and observables } \\ & 13\end{array}$

$\begin{array}{ll}3.4 & \text { Examples: } n=6 \text { and } n=9\end{array}$

$\begin{array}{lll}3.5 & \text { Extension to more than one neutrino field } & 17\end{array}$

$\begin{array}{llr}4 \text { Conclusions } & 19\end{array}$

A Treatment of the model as a single-field inflation model 20

B Derivation of the equation of motion in the effective single-field inflation

$\begin{array}{ll}\text { framework } & 23\end{array}$

\section{Introduction}

Following the discovery of neutrino oscillations [1], we know that neutrinos have small masses with large mixing. In the absence of a signal of neutrinoless double beta decay, the nature of neutrino mass is unknown: they could be either Dirac or Majorana. ${ }^{1}$ In the Standard Model (SM), where neutrinos are massless, the Lagrangian has an accidental global $\mathrm{U}(1)_{L}$ symmetry, corresponding to lepton number $L$ being conserved. ${ }^{2}$ If Majorana neutrino masses are introduced, such terms would explicitly break lepton number $L$ by two units. The origin of such light Majorana neutrino masses is unknown but it clearly is related to the question of the breaking of $L$. In general $L$ may be broken explicitly or spontaneously [2]. A commonly considered possibility for the origin of Majorana neutrino masses is via the effective Weinberg operator [3] which explicitly breaks $L$ by two units. In the typeI seesaw model [4-9], the origin of the effective Weinberg operator is due to right-handed Majorana neutrino masses, which would also break $L$ by two units. The origin of such right-handed neutrino masses is therefore also related to the breaking of lepton number.

\footnotetext{
${ }^{1}$ It is also possible that both Dirac and Majorana mass terms could appear together in the Lagrangian.

${ }^{2}$ It also respects baryon number $B$, corresponding to an accidental global $\mathrm{U}(1)_{B}$ symmetry.
} 
In the framework of the type-I seesaw mechanism, one possibility is to have righthanded neutrino mass terms appearing explicitly in the Lagrangian, which would correspond to explicitly break lepton number. This could be called "soft breaking" of $L$ since such mass terms have dimension 3 , which is less than 4 . Another possibility is that $L$ is an exact global symmetry which is spontaneously broken by some scalar field $\Phi$, resulting in right-handed neutrino masses and a Goldstone boson called the Majoron [10]. The idea is to consider a scalar $\Phi$ with $L=2$ giving mass to the right-handed neutrinos $N^{c}$ via the Yukawa interaction

$$
\mathcal{L}_{\Phi N N}=-\lambda \Phi \overline{N_{R}^{c}} N_{R}+\text { H.c. }
$$

Since $\Phi$ acquiring a vev $\langle\Phi\rangle \neq 0$ breaks lepton number conservation, one may call $\Phi$ the Majoron field, although more commonly this name is reserved for the associated Goldstone boson which arises when the global $\mathrm{U}(1)_{L}$ symmetry is spontaneously broken. This model is often referred to as the singlet Majoron model, and we shall refer to the complex scalar field $\Phi$ as the Majoron field.

A supersymmetric (SUSY) singlet Majoron extension of the minimal supersymmetric standard model (MSSM) has also been proposed [11] and studied [12-15]. The main focus of these studies has been on spontaneous $R$-parity violation. However, we note that, in all singlet Majoron models, it seems unlikely that $L$ would be an exact global symmetry, since global symmetries are not as protected as gauge symmetries. It seems more likely that in all such Majoron models, supersymmetric or not, the lepton number $L$ would be an approximate global symmetry, broken by some higher order operators. An exception would be models where the anomaly-free combination $\mathrm{U}(1)_{B-L}$ is gauged, but in this paper we shall not consider this possibility.

In this paper we consider a supersymmetric model in which global lepton number $L$ is explicity broken by higher order terms of the form $\Phi^{n}$ in the superpotential and potential. Such terms explicitly break global $\mathrm{U}(1)_{L}$ down to a discrete subgroup of lepton number $\mathbb{Z}_{N}$, where $N=n$ if $n$ is odd and $N=2 n$ if $n$ is even. The resulting scalar potential (calculated in the framework of supergravity) leads to a vacuum expectation value of $\Phi$ which spontaneously breaks the discrete lepton number $\mathbb{Z}_{N}$. We shall focus on the possibility that, before spontaneous symmetry breaking, the scalar potential, including non-minimal Kähler corrections, is suitable for cosmological inflation [16, 17]. This is interesting since it relates inflation to the mechanism responsible for the origin of neutrino masses. In particular, the complex Majoron field $\Phi$ simultaneously provides inflation and, at the end of inflation at the global minimum, right-handed neutrino masses. Majoron inflation has been studied recently in [18], without supersymmetry, although higher order $\Phi^{n}$ terms were not considered and large tensor modes were shown to result from chaotic type inflation. ${ }^{3}$ By contrast, here we shall use the higher order $\Phi^{n}$ terms to generate a model of new inflation (or "hilltop inflation") very similar to the one presented in [21] (see also [22-28]), i.e. the framework will be supergravity with non-minimal Kähler potential, leading to small tensor modes. It is also interesting to note that in our model the Majoron

\footnotetext{
${ }^{3} \mathrm{~A}$ very similar scenario has been studied in [19, 20], where inflation is driven by a mixture of the Majoron and the SM Higgs field.
} 


\begin{tabular}{|c|ccccc|}
\hline & $\hat{X}_{0}$ & $\hat{\Phi}$ & $\hat{N}^{c}$ & $\hat{H}_{u}$ & $\hat{L}$ \\
\hline $\mathbb{Z}_{N}$ & 0 & 2 & -1 & 0 & 1 \\
\hline$R$ & 2 & 0 & 1 & 0 & 1 \\
\hline
\end{tabular}

Table 1. The lepton $\hat{L}$, Higgs $\hat{H}_{u}$, CP conjugated right-handed neutrino $\hat{N}^{c}$, auxiliary $\hat{X}_{0}$ and inflaton (Majoron) $\hat{\Phi}$ superfields of the model, and how they transform under the symmetries $\mathbb{Z}_{N}$ and $\mathrm{U}(1)_{R}$.

field $\Phi$ may also carry flavour symmetry quantum numbers and may be considered also to be a flavon, as for example in the model in [29], where different right-handed neutrinos carry different $\mathbb{Z}_{N}$ charges. Flavon inflation has for example been considered in [30-32]. There have also been other approaches which attempt to relate inflation to neutrino masses, for example, our Lagrangian is similar to the one of [33] where, however, the sneutrino component of $N^{c}$ is used as the inflaton, while we use the Majoron scalar field $\Phi$. Further approaches to relating inflation to neutrino masses have also been considered [34-40].

The outline of this paper is as follows. In section 2 we discuss the structure of our model and the resulting scalar potential in the framework of supergravity with a non-minimal Kähler potential. In section 3 we investigate how to avoid the domain wall problem in our model and discuss the phenomenology of inflation. There we also provide plots of the allowed parameter space of our model for a selection of cutoff-scales $\Lambda$ and values $n$ in the higher-dimensional operator $\Phi^{n} / \Lambda^{n-2}$ in the superpotential. Finally, we present our conclusions in section 4 .

\section{The model with non-minimal Kähler potential}

We consider a superpotential involving five superfields: an auxiliary field $\hat{X}_{0}$, the inflaton (Majoron) field $\hat{\Phi}$, a CP conjugated right-handed neutrino field $\hat{N}^{c}$, the Higgs field $\hat{H}_{u}$ and a lepton field $\hat{L}$. The Higgs and Lepton doublets have usual Standard Model (SM) gauged electroweak quantum numbers, while the other superfields above are SM singlets. We impose a discrete symmetry $\mathbb{Z}_{N}$ under which $\hat{X}_{0}$ and $\hat{H}_{u}$ carry zero charge, while $\hat{\Phi}$ has charge $2, \hat{N}^{c}$ has charge -1 , and $\hat{L}$ has charge +1 , as shown in table 1 . It is clear from table 1 that $\mathbb{Z}_{N}$ can be thought of as a discrete subgroup of lepton number $\mathrm{U}(1)_{L}$.

In addition, we assume an $R$ symmetry, with charge assignments also shown in table 1. The lowest order superpotential with $R=2$ allowed by the symmetries is then given by ${ }^{4}$

$$
\mathcal{W}=\hat{X}_{0}\left(\frac{\hat{\Phi}^{n}}{\Lambda^{n-2}}-M^{2}\right)+\lambda \hat{\Phi} \hat{N}^{c} \hat{N}^{c}+y \hat{L} \hat{H}_{u} \hat{N}^{c} .
$$

\footnotetext{
${ }^{4}$ If we include also the Higgs field $\hat{H}_{d}$, there will be an additional term $\hat{X}_{0} \hat{H}_{u} \hat{H}_{d}$ in the superpotential. As we will see later, the Kähler potential of the model can always be chosen in such a way that all scalar fields apart from the scalar component of $\hat{\Phi}$ vanish during inflation. Therefore, we do not need to take this additional coupling into account in this paper and we will only consider couplings which involve the inflaton or the neutrino field. Note that the $R$ symmetry forbids the $\mu$-term $\mu \hat{H}_{u} \hat{H}_{d}$. An effective $\mu$-term could be generated from the coupling $\hat{X}_{0} \hat{H}_{u} \hat{H}_{d}$ as described in [41].
} 
For even $N$, the lowest power of $\hat{\Phi}$ which couples to $\hat{X}_{0}$ in the superpotential is given by $\hat{\Phi}^{n}$ with $n=N / 2$, while for odd $N$ we have $n=N$. When the real scalar component of $\hat{\Phi}$ develops a vacuum expectation value (vev), this will break the $\mathbb{Z}_{N}$ symmetry completely if $N$ is odd, or it will preserve a $\mathbb{Z}_{2}$ subgroup of $\mathbb{Z}_{N}$ if $N$ is even.

The mass parameters $\Lambda, M$, and the dimensionless couplings $\lambda$ and $y$ can all be made real and positive by rephasing of $\hat{\Phi}, \hat{N}^{c}, \hat{X}_{0}$ and $\hat{L}$, i.e. without loss of generality we may assume

$$
\Lambda>0, \quad M^{2}>0, \quad \lambda>0, \quad y>0 .
$$

We start with the minimal Kähler potential

$$
\mathcal{K}=\left|\hat{X}_{0}\right|^{2}+|\hat{\Phi}|^{2}+\left|\hat{N}^{c}\right|^{2}+\left|\hat{H}_{u}\right|^{2}+|\hat{L}|^{2} .
$$

The F-term scalar potential is then given by

$$
\begin{aligned}
V & =e^{\mathcal{K} / m_{\mathrm{Pl}}^{2}}\left\{\sum_{i, j}\left(K^{-1}\right)_{i j} D_{z_{i}} \mathcal{W}\left(D_{z_{j}} \mathcal{W}\right)^{*}-3 m_{\mathrm{Pl}}^{-2}|\mathcal{W}|^{2}\right\}, \\
D_{z_{i}} \mathcal{W} & =\partial_{z_{i}} \mathcal{W}+m_{\mathrm{Pl}}^{-2} \mathcal{W} \partial_{z_{i}} \mathcal{K}, \quad K_{i j}=\frac{\partial^{2} \mathcal{K}}{\partial z_{i} \partial z_{j}^{*}},
\end{aligned}
$$

where $z_{i}=X_{0}, \Phi, N^{c}, H_{u}, L .^{5}$ The reduced Planck mass $m_{\mathrm{Pl}}=2.435 \times 10^{18} \mathrm{GeV}$ is related to Newton's constant via $m_{\mathrm{Pl}}^{-2} \equiv 8 \pi G$. The D-term contributions to the scalar potential are at least quartic in the fields, so they do not contain any mass terms of fields. Consequently, when we will discuss the masses of the fields below, we can neglect the Dterm contributions. At the end of this discussion it will turn out that it is possible to choose the Kähler potential in such a way that all fields apart from $\Phi$ are zero during inflation. As a consequence, since the D-term contributions are at least bilinear in the gauge multiplet fields, they vanish during (and after) inflation. Therefore, for our purposes, it is sufficient to study the F-term scalar potential, i.e. $V=V_{F}$ in this paper.

Let us now study the prerequisites for slow-roll inflation by computing the masses of the involved fields. To do so, we reformulate $V$ in terms of the ten real fields $\varphi=$ $\left(\operatorname{Re} X_{0}, \operatorname{Im} X_{0}, \operatorname{Re} \Phi, \operatorname{Im} \Phi, \operatorname{Re} N^{c}, \operatorname{Im} N^{c}, \operatorname{Re} H_{u}, \operatorname{Im} H_{u}, \operatorname{Re} L, \operatorname{Im} L\right)$. The bilinear terms in the fields are then given by

$$
\mathcal{L}_{2}=\left.\frac{1}{2} \sum_{i, j=1}^{10}\left(\mathcal{M}^{2}\right)_{i j} \varphi_{i} \varphi_{j} \equiv \frac{1}{2} \sum_{i, j=1}^{10} \frac{\partial^{2} V(\varphi)}{\partial \varphi_{i} \partial \varphi_{j}}\right|_{\varphi=0} \varphi_{i} \varphi_{j}
$$

The squared-mass matrix $\mathcal{M}^{2}$ for our model is given by

$$
\mathcal{M}^{2}=\frac{2 M^{4}}{m_{\mathrm{Pl}}^{2}} \operatorname{diag}(0,0,1,1,1,1,1,1,1,1),
$$

i.e. with the minimal Kähler potential the real and imaginary parts of $X_{0}$ remain massless, while all other fields have a mass $\sqrt{2} M^{2} / m_{\mathrm{Pl}}$. In new inflation models the fields

\footnotetext{
${ }^{5}$ We denote the scalar component of a superfield $\hat{\phi}$ by $\phi$. In particular, $z_{i}$ are the complex scalar components.
} 
are assumed to have small values (usually smaller than $m_{\mathrm{Pl}}$ ), such that the potential is dominated by the constant term

$$
V_{0}=V(0)=M^{4}
$$

In the slow-roll approximation (which we require to be valid during inflation) the Hubble constant is determined by

$$
H^{2} \approx \frac{V}{3 m_{\mathrm{Pl}}^{2}}
$$

which in our case becomes

$$
H^{2} \approx \frac{V_{0}}{3 m_{\mathrm{Pl}}^{2}}=\frac{M^{4}}{3 m_{\mathrm{Pl}}^{2}} .
$$

Fields with masses greater than the Hubble parameter rapidly evolve to their minimum and are therefore not capable of creating a long enough exponential expansion of the Universe. In our simple model we have

$$
\begin{aligned}
m_{\operatorname{Re} \Phi}^{2} & =m_{\operatorname{Im} \Phi}^{2}=m_{\operatorname{Re} N^{c}}^{2}=m_{\operatorname{Im} N^{c}}^{2}= \\
m_{\operatorname{Re} H_{u}}^{2} & =m_{\operatorname{Im} H_{u}}^{2}=m_{\operatorname{Re} L}^{2}=m_{\operatorname{Im} L}^{2}=\frac{2 M^{4}}{m_{\mathrm{Pl}}^{2}}>H^{2},
\end{aligned}
$$

so none of the components of $\Phi, N^{c}, H_{u}$ and $L$ can be the inflaton. Since, however, we are interested in a model based on $\Phi$ as the inflaton, we have to modify the potential.

Motivated by our desire for $\Phi$ to be the inflaton, we consider a non-minimal Kähler potential $[21,30-33]$ for the five superfields $\hat{X}_{0}, \hat{\Phi}, \hat{N}^{c}, \hat{H}_{u}$ and $\hat{L}$ which to order $m_{\mathrm{Pl}}^{-2}$ has the form, ${ }^{6}$

$$
\mathcal{K}=\sum_{i}\left|\hat{S}_{i}\right|^{2}+\frac{1}{m_{\mathrm{Pl}}^{2}} \sum_{i<j} \kappa_{i j}\left|\hat{S}_{i}\right|^{2}\left|\hat{S}_{j}\right|^{2}+\frac{1}{m_{\mathrm{Pl}}^{2}} \sum_{i} \kappa_{i}\left|\hat{S}_{i}\right|^{4}
$$

where $\hat{S}_{i}=\hat{X}_{0}, \hat{\Phi}, \hat{N}^{c}, \hat{H}_{u}, \hat{L}$. Computing $V$ as before, $V_{0}=M^{4}$ is unchanged, but the squared masses become

$$
m_{\operatorname{Re} X_{0}}^{2}=m_{\operatorname{Im} X_{0}}^{2}=-\frac{8 \kappa_{X_{0}} M^{4}}{m_{\mathrm{Pl}}^{2}}
$$

and

$$
m_{\operatorname{Re} S}^{2}=m_{\mathrm{Im} S}^{2}=\frac{2 M^{4}}{m_{\mathrm{Pl}}^{2}}\left(1-\kappa_{X_{0} S}\right) \quad\left(S=\Phi, N^{c}, H_{u}, L\right) .
$$

We want the scalar field $\Phi$ (the Majoron) to be the inflaton. This can lead to successful inflation since the potential involving $\Phi$ is particularly flat due to its high power $n$ in the scalar potential. In order to achieve this we need to ensure that, locally, all scalar fields apart from $\Phi$ have large enough positive mass squares so that they quickly roll to their zero field values, while $\Phi$ has a negative mass squared, and slowly rolls away from its zero field value. This is sometimes referred to as "hilltop" inflation.

\footnotetext{
${ }^{6}$ The $\mathbb{Z}_{N}$-symmetry of the model and the requirement of a real Kähler potential would also allow adding terms of the form $\kappa_{n} m_{\mathrm{Pl}}^{2-n}\left(\hat{\Phi}^{n}+\hat{\Phi}^{* n}\right)$. This, however, will not be needed to tune the masses of the fields.
} 
In order to achieve this we suppose that, for all fields apart from $\Phi$,

$$
\kappa_{X_{0}}<-\frac{1}{24} \quad \text { and } \quad \kappa_{X_{0} S}<\frac{5}{6} \quad(S \neq \Phi) .
$$

Then all fields except $\Phi$ will have masses larger than $H$ such that they rapidly evolve to their minima. If the conditions in eq. (2.15) are satisfied, the squared-mass matrix at zero field value is positive definite for all fields except $\Phi$ and

$$
\left.\frac{\partial V}{\partial \varphi_{i}}\right|_{\varphi_{i}=0}=0 \quad \forall \varphi_{i}
$$

Consequently, the minimum of $V$ the fields $S \neq \Phi$ will rapidly evolve to is $S=0$. Therefore, we can set $X_{0}=N^{c}=H_{u}=L=0$ during inflation.

Turning to the field $\Phi$ itself, we shall choose

$$
\kappa_{X_{0} \Phi}>1
$$

so that the field $\Phi$ gets a negative mass term making $\Phi=0$ a local maximum of $V$. This allows inflation with $\Phi$ slowly rolling to a local minimum at $\Phi \neq 0$.

Assuming $X_{0}=N^{c}=H_{u}=L=0$ during inflation, the relevant potential for the complex scalar field $\Phi$ becomes, using eq. (2.4), with eqs. (2.1) and (2.12),

$$
V(\Phi)=\frac{\exp \left\{\frac{|\Phi|^{2}}{m_{\mathrm{Pl}}^{2}}\left(1+\kappa_{\Phi} \frac{|\Phi|^{2}}{m_{\mathrm{Pl}}^{2}}\right)\right\}}{1+\kappa_{X_{0}} \Phi \frac{|\Phi|^{2}}{m_{\mathrm{P} 1}^{2}}}\left|\frac{\Phi^{n}}{\Lambda^{n-2}}-M^{2}\right|^{2} .
$$

The Lagrangian for $\Phi$ is then given by

$$
\begin{aligned}
\mathcal{L}(\Phi) & =\left.\frac{\partial^{2} \mathcal{K}}{\partial \Phi \partial \Phi^{*}}\right|_{X_{0}=N^{c}=H_{u}=L=0}\left(\partial_{\mu} \Phi\right)\left(\partial^{\mu} \Phi\right)^{*}-V(\Phi) \\
& =\left(1+4 \kappa_{\Phi} \frac{|\Phi|^{2}}{m_{\mathrm{Pl}}^{2}}\right)\left(\partial_{\mu} \Phi\right)\left(\partial^{\mu} \Phi\right)^{*}-V(\Phi) .
\end{aligned}
$$

The assumption of a non-minimal Kähler potential thus leads to a non-canonically normalized kinetic term in the Lagrangian. However, since the effects of a non-minimal Kähler potential on reheating are irrelevant, only the spectral index $n_{S}$, the tensor-to-scalar ratio $r$ and the running of the spectral index may be subject to relevant contributions from a non-minimal $\mathcal{K}$. Since it turns out that our model can easily comply with the observed values/bounds on these quantities for $\kappa_{\Phi}=0$, we may avoid the complication of non-canonical normalization by assuming $\kappa_{\Phi}$ to be small enough to neglect its effect in the kinetic terms. Therefore, in the remainder of the paper we will assume canonically normalized kinetic terms, i.e.

$$
\mathcal{L}(\Phi)=\left(\partial_{\mu} \Phi\right)\left(\partial^{\mu} \Phi\right)^{*}-V(\Phi)
$$

with the potential $V$ of eq. (2.18).

Since our inflation model is supersymmetric, $\Phi$ is necessarily complex, and one might think that we would need to treat the model as a two-field inflation model, with the two real fields being $\operatorname{Re} \Phi$ and $\operatorname{Im} \Phi$. However, it is possible to show that, during the inflationary 
epoch, the ratio $\operatorname{Im} \Phi / \operatorname{Re} \Phi$ is effectively frozen, with inflationary dynamics controlled by the magnitude of the complex Majoron field $|\Phi|$. This is explained in detail in appendices A and B. The result is a set of equations of motion for a single inflaton field $\phi \equiv \sqrt{2}|\Phi|$ which reads

$$
\begin{aligned}
& \ddot{\phi}+3 H \dot{\phi}+\frac{\partial V(\phi, \psi)}{\partial \phi}=0, \\
& H^{2}=\frac{1}{3 m_{\mathrm{Pl}}^{2}}\left(V+\frac{1}{2} \dot{\phi}^{2}\right),
\end{aligned}
$$

where $\psi=\operatorname{Arg} \Phi=\arctan (\operatorname{Im} \Phi / \operatorname{Re} \Phi)$ and the derivative has to be evaluated at the approximately constant value $\psi_{0}$ during inflation.

\section{Majoron inflation}

We now have the relevant prerequisites in order to study the domain wall problem and the phenomenology of single field Majoron inflation in our model, which we will do in this section, where the form of the potential $V(\phi, \psi)$ will be discussed.

\subsection{The domain wall problem}

In this subsection, we first discuss the conditions for avoiding the domain wall problem [42] in our model. The presently (and in the future) observable Universe originates from a small patch of the pre-inflationary Universe with homogenous initial conditions $\Phi\left(t_{0}\right)$ in the whole patch. Since during inflation there is an immense drop in temperature, thermal fluctuations will not affect the time evolution of $\Phi$. Consequently, $\Phi$ will approach the same minimum everywhere in the Universe, therefore not forming domains during inflation. The crucial question is whether in the reheating phase of the Universe, the temperature $T_{R}$ reaches a value higher than the potential barrier between the $n$ equivalent minima of the $\mathbb{Z}_{n}$-symmetric potential (2.18). To answer this question, we have to compute the height of the barrier and the reheating temperature, which we will do in this section. We will not discuss creation of domain walls due to quantum fluctuations in this paper.

\subsubsection{The height of the barrier}

Reformulating the scalar potential (2.18) in terms of two real and positive fields $\phi$ and $\psi$ defined as

$$
\Phi=\frac{1}{\sqrt{2}}\left(\phi_{R}+i \phi_{I}\right) \equiv \frac{1}{\sqrt{2}} \phi e^{i \psi}
$$

we obtain

$$
V(\phi, \psi)=f(\phi) g(\phi, \psi)
$$

with

$$
f(\phi)=\frac{\exp \left\{\frac{\phi^{2}}{4 m_{\mathrm{Pl}}^{2}}\left(2+\kappa_{\Phi} \frac{\phi^{2}}{m_{\mathrm{Pl}}^{2}}\right)\right\}}{2^{n} \Lambda^{2 n-4}\left(1+\kappa_{X_{0} \Phi} \frac{\phi^{2}}{2 m_{\mathrm{Pl}}^{2}}\right)}, \quad g(\phi, \psi)=\left(\phi^{n}-v_{M}^{n}\right)^{2}+2 v_{M}^{n} \phi^{n}(1-\cos (n \psi)),
$$


where

$$
v_{M} \equiv \sqrt{2}\left(M^{2} \Lambda^{n-2}\right)^{1 / n}
$$

is the vev of $\phi$. The height of the barrier between two minima $(\cos (n \psi)=1)$ is thus given by

$$
4 f(\phi) v_{M}^{n} \phi^{n}
$$

Since $\phi \ll m_{\mathrm{Pl}}$, we can expand $f(\phi)$ in $m_{\mathrm{Pl}}^{-1}$ giving

$$
f(\phi)=\frac{1}{2^{n} \Lambda^{2 n-4}}\left(1-\beta \frac{\phi^{2}}{m_{\mathrm{Pl}}^{2}}\right)+\mathcal{O}\left(m_{\mathrm{Pl}}^{-4}\right),
$$

where

$$
\beta \equiv \frac{\kappa_{X_{0} \Phi}-1}{2}>0
$$

The height of the barrier is therefore given by

$$
\Delta V(\phi) \approx \frac{v_{M}^{n} \phi^{n}}{2^{n-2} \Lambda^{2 n-4}}=\frac{\sqrt{2}^{4-n} M^{2}}{\Lambda^{n-2}} \phi^{n} .
$$

\subsubsection{The reheat temperature}

We estimate the reheat temperature $T_{R}$ using the prescription of [43]. For our purposes we only need to know the order of magnitude of the reheat temperature and, therefore, it is sufficient to treat also reheating as if our model was a single-field inflation model. For our computation we assume that the system at the beginning of reheating already has evolved to one of its minima with respect to $\psi=\operatorname{Arg} \Phi$, in which case the potential (3.2) becomes ${ }^{7}$

$$
V(\phi)=f(\phi)\left(\phi^{n}-v_{M}^{n}\right)^{2} \approx \frac{\left(\phi^{n}-v_{M}^{n}\right)^{2}}{2^{n} \Lambda^{2 n-4}},
$$

with $v_{M}$ defined in eq. (3.4). The equation of motion for $\phi$ can then be recast as

$$
\begin{aligned}
& \ddot{\phi}+3 H \dot{\phi}+\frac{\partial V}{\partial \phi}=0, \\
& H^{2}=\frac{1}{3 m_{\mathrm{Pl}}^{2}}\left(V+\frac{1}{2} \dot{\phi}^{2}\right),
\end{aligned}
$$

which is explained in detail in appendix B.

Reheating happens through the decay of coherent oscillations of the inflaton field (inflaton particles) to other particles which subsequently thermalize. The equation of motion then contains an additional friction term [43] proportional to the decay width $\Gamma_{\phi}$ of the inflaton:

$$
\ddot{\phi}+3 H \dot{\phi}+\Gamma_{\phi} \dot{\phi}+\frac{\partial V}{\partial \phi}=0 .
$$

Reheating therefore begins when the decay rate becomes comparable to the expansion rate of the Universe, i.e. for $\Gamma_{\phi} \gtrsim H$. Inflaton decay proceeds via the Yukawa coupling

$$
-\lambda \Phi \overline{N_{R}^{c}} N_{R}+\text { H.c. }
$$

\footnotetext{
${ }^{7}$ Note that in the minima with respect to $\psi$ one has $\Phi^{n}=\phi^{n} / \sqrt{2}^{n}$.
} 
to the right-handed neutrinos. Assuming $m_{N} \ll m_{\text {Inf }}$ the rate for the decay into (s)neutrinos is given by ${ }^{8}$

$$
\Gamma_{\phi}=\frac{\lambda^{2} m_{\mathrm{Inf}}}{16 \pi}
$$

where $m_{\text {Inf }}$ is the inflaton mass.

Reheating starts at $\Gamma_{\phi} \sim H$ which implies

$$
V+\frac{1}{2} \dot{\phi}^{2}=3 m_{\mathrm{Pl}}^{2} H^{2} \sim \frac{3 m_{\mathrm{Pl}}^{2} \lambda^{4} m_{\mathrm{Inf}}^{2}}{256 \pi^{2}} .
$$

The left-hand side of this equation is just the energy density of the scalar field. Assuming that once reheating starts it is almost completely converted into thermal energy of the decay products, we find

$$
\frac{g_{*} \pi^{2}}{30} T_{R}^{4} \sim \frac{3 m_{\mathrm{Pl}}^{2} \lambda^{4} m_{\mathrm{Inf}}^{2}}{256 \pi^{2}} .
$$

The reheat temperature is thus given by

$$
T_{R}^{4} \sim \frac{45 m_{\mathrm{Pl}}^{2} \lambda^{4} m_{\mathrm{Inf}}^{2}}{128 \pi^{4} g_{*}}
$$

where $g_{*}$ is the number of (ultrarelativistic) degrees of freedom of the thermal bath created by reheating. ${ }^{9}$

\subsubsection{Creation of domain walls after inflation}

We finally want to compare the reheat temperature to the height of the barrier between two minima during the reheating process. For this we need the inflaton mass, which is obtained from the squared-mass matrix

$$
\left(M_{\phi}^{2}\right)_{i j}=\left.\frac{\partial^{2} V}{\partial \phi_{i} \partial \phi_{j}}\right|_{i, j=R, I ; \phi=v_{M}}=n^{2} M^{2}\left(\frac{M}{\Lambda}\right)^{2\left(1-\frac{2}{n}\right)} \delta_{i j}
$$

at the global minimum $\phi=v_{M}$ of the potential. The inflaton mass is thus given by

$$
m_{\mathrm{Inf}}=n M\left(\frac{M}{\Lambda}\right)^{1-\frac{2}{n}}
$$

Note that in our model the masses of the scalar field $\phi$ and the pseudoscalar field $\psi$ are both equal to $m_{\text {Inf }}$ at the global minimum of the potential. Thus, there are two degenerate physical particles with common mass $m_{\phi}=m_{\psi}=m_{\text {Inf }}$, which may be observable in future collider experiments, if $m_{\mathrm{Inf}}$ is low enough. The other information we need is the height

\footnotetext{
${ }^{8}$ The textbook formula for a 2-body decay has $8 \pi$ in the denominator. Here we have identical fermions/scalars in the final state, which yields an additional factor $1 / 2$. Another factor $1 / 2$ comes from the fact that only the right-handed components of the neutrinos couple to the inflaton. Finally, there is a factor 2 , since also the decay into sneutrinos is possible.

${ }^{9}$ Even if the mass of the right-handed (s)neutrinos is larger than the reheat temperature a thermal bath can be created due to the decay of the (s)neutrinos via the Yukawa coupling $y L H_{u} N^{c}$.
} 
$\Delta V$ of the potential barrier at the beginning of reheating. From eq. (3.14) we see that at the beginning of reheating

$$
V<\frac{3 m_{\mathrm{Pl}}^{2} \lambda^{4} m_{\mathrm{Inf}}^{2}}{256 \pi^{2}}
$$

which, using approximation (3.9), yields

$$
\phi^{n}>v_{M}^{n}-\frac{\sqrt{3 \times 2^{n}} \lambda^{2} \Lambda^{n-2} m_{\mathrm{Pl}} m_{\mathrm{Inf}}}{16 \pi}
$$

i.e.

$$
\Delta V>4 M^{4}-\frac{n \sqrt{3} \lambda^{2}}{4 \pi}\left(\frac{M}{\Lambda}\right)^{1-\frac{2}{n}} m_{\mathrm{Pl}} M^{3} .
$$

The creation of domain walls due to the thermal energy released by the reheating process will be suppressed as long as

$$
\frac{\Delta V^{1 / 4}}{T_{R}}>1
$$

and thus the requirement for avoiding domain wall creation in our model is

$$
\frac{\Delta V}{T_{R}^{4}}=\frac{4\left(\frac{M}{m_{\mathrm{Pl}}}\right)^{2}-\frac{n \sqrt{3}}{4 \pi} \frac{M}{m_{\mathrm{Pl}}} a}{\frac{45 n^{2}}{128} \frac{1}{\pi^{4} g_{*}} a^{2}} \gg 1,
$$

where we have introduced the abbreviation

$$
a \equiv \lambda^{2}\left(\frac{M}{\Lambda}\right)^{1-\frac{2}{n}}
$$

In the limit ${ }^{10}$

$$
\frac{n \sqrt{3}}{4 \pi} \frac{M}{m_{\mathrm{Pl}}} a \gg \frac{45 n^{2}}{128} \frac{1}{\pi^{4} g_{*}} a^{2} \Rightarrow \frac{m_{\mathrm{Pl}}}{M} a \ll \frac{32 \pi^{3} \sqrt{3}}{45 n} g_{*},
$$

the condition (3.23) simplifies to

$$
4\left(\frac{M}{m_{\mathrm{Pl}}}\right)^{2} \gg \frac{n \sqrt{3}}{4 \pi} \frac{M}{m_{\mathrm{Pl}}} a
$$

or

$$
\frac{m_{\mathrm{Pl}}}{M} a \ll \frac{16 \pi}{n \sqrt{3}} \approx \frac{30}{n} .
$$

The condition for avoiding the domain wall problem in our model is thus given by

$$
\lambda^{2}\left(\frac{M}{\Lambda}\right)^{1-\frac{2}{n}} \frac{m_{\mathrm{Pl}}}{M} \ll \frac{16 \pi}{n \sqrt{3}}
$$

or

$$
\lambda^{2} \ll \frac{16 \pi}{n \sqrt{6}} \frac{v_{M}}{m_{\mathrm{Pl}}} .
$$

Therefore, the condition to avoid domain walls provides a bound on the Yukawa coupling of the Majoron field to the right-handed neutrinos. Interestingly, this bound depends only on the vev $v_{M}$ of the Majoron.

\footnotetext{
${ }^{10}$ We assume the reheating temperature to be higher than the top-quark mass, yielding the lower bound $g_{*}>\left(g_{*}\right)_{\mathrm{SM}}=106.75$. Therefore, the right-hand side of inequality $(3.25)$ is larger than $\mathcal{O}(1000) / n$. Comparison to the finally obtained bound on $a$ - see eq. (3.27) - thus justifies this approximation.
} 


\subsection{Inflation phenomenology}

In order to compute the CMB observables, we need to compute the slow-roll parameters which are given by

$$
\begin{aligned}
\epsilon & =\frac{1}{2} m_{\mathrm{Pl}}^{2}\left(\frac{V^{\prime}}{V}\right)^{2}, \\
\eta & =m_{\mathrm{Pl}}^{2} \frac{V^{\prime \prime}}{V}, \\
\xi & =m_{\mathrm{Pl}}^{4} \frac{V^{\prime} V^{\prime \prime \prime}}{V^{2}},
\end{aligned}
$$

where ${ }^{\prime}=\partial / \partial \phi$. The potential is given by eqs. (3.2) and (3.3), where $\psi=\psi_{0}$, the approximately constant value of the phase $\psi$ during inflation. In the following we will show that

$$
\epsilon \ll 1 \text {. }
$$

In order to show this, we first show that during inflation $\phi \ll v_{M}$. The slow-roll parameter $\eta$ for $\phi \ll v_{M}$ is given by

$$
\eta \simeq-\frac{2 n(n-1) \phi^{n-2} m_{\mathrm{Pl}}^{2} \cos \left(n \psi_{0}\right)}{v_{M}^{n}}-2 \beta+\mathcal{O}\left(m_{\mathrm{Pl}}^{-2}\right),
$$

where $\beta$ is defined in eq. (3.7). During inflation we must have $|\eta|<1$ which necessarily implies

$$
\left|A \phi^{n-2}+2 \beta\right|<1
$$

where we have defined

$$
A \equiv \frac{2 n(n-1) m_{\mathrm{Pl}}^{2} \cos \left(n \psi_{0}\right)}{v_{M}^{n}} .
$$

If $\beta$ is not much larger than $\mathcal{O}(1)$ and $\cos \left(n \psi_{0}\right)$ is not accidentally close to zero, this leads to the upper bound

$$
\frac{\phi}{v_{M}} \lesssim\left(\frac{v_{M}^{2} / m_{\mathrm{Pl}}^{2}}{2 n(n-1)}\right)^{\frac{1}{n-2}} .
$$

This inequality necessarily holds also for $\phi_{R}$ and $\phi_{I}$ and thus

$$
\left|\phi_{R}\right|^{n-2} \lesssim \frac{1}{2(n-1)} \frac{v_{M}^{n}}{n m_{\mathrm{Pl}}^{2}}
$$

Comparing this equation to the condition (A.18) leads to the conclusion that the evolution of the ratio $\operatorname{Im} \Phi / \operatorname{Re} \Phi$ is always frozen in our model during inflation, and the assumption of effective single-field inflation is justified.

Since $v_{M}$ as the vev of $\phi$ is the flavour symmetry breaking scale, we assume that $v_{M} \lesssim M_{\mathrm{GUT}} \sim 10^{-3} m_{\mathrm{Pl}}$, in which case we find

$$
n=3: \phi \lesssim 10^{-7} v_{M}, \quad n=6: \phi \lesssim 10^{-2} v_{M}, \quad n=9: \phi \lesssim 0.07 v_{M}
$$

Therefore, for moderate $n$, e.g. $n=6$, as anticipated $\phi \ll v_{M}$. 
In the limit $\phi \ll v_{M} \ll m_{\mathrm{Pl}}$ the potential is given by ${ }^{11}$

$$
V\left(\phi, \psi_{0}\right) \simeq M^{4}\left(1-\beta \frac{\phi^{2}}{m_{\mathrm{Pl}}^{2}}-2 \frac{\phi^{n}}{v_{M}^{n}} \cos \left(n \psi_{0}\right)\right)
$$

This shows the recognisable "hilltop" form of the potential, corrected by a Planck scale suppressed term proportional to the parameter $\beta$. Inserting the approximate expression for $V$ into the definitions of the slow-roll parameters, and using the approximation $V \approx M^{4}$ during inflation, one finds

$$
\epsilon=2\left(\beta \frac{\phi}{m_{\mathrm{Pl}}}+n \frac{\phi^{n-1} m_{\mathrm{Pl}}}{v_{M}^{n}} \cos \left(n \psi_{0}\right)\right)^{2} .
$$

For $\beta=1, \cos \left(n \psi_{0}\right)=1, v_{M}=10^{-3} m_{\mathrm{Pl}}$ and the upper bound (3.35) for $\phi$ one finds $\epsilon \sim 10^{-8}$ for $n=9$ and much smaller values for smaller $n$, i.e. $\epsilon$ effectively vanishes in our model. Therefore, one prediction of our model is an unobservably small tensor to scalar ratio $r=16 \epsilon$.

In order to compute the spectral index, we need to know the field value $\phi_{e}$ at the end of inflation. Since $\epsilon$ is negligibly small, slow-roll inflation may end either at $|\eta|=1$ or $|\xi|=1$. However, it is possible to show that there is a minimal value of $\beta$ for which $|\xi|$ can reach the value 1 , which is given by

$$
\beta=\frac{1}{\sqrt{(n-1)(n-2)}}
$$

It will turn out that, in order to reproduce the correct spectral index, $\beta$ must be (much) smaller than 0.1 , so for $n$ smaller than $12,|\xi|<1$ and the end of inflation is characterized by $|\eta|=1$. Restricting ourselves to $\beta<1 / 2$ this gives

$$
\begin{aligned}
\phi_{e}^{n-2} & =\frac{1-2 \beta}{A} \quad\left(\cos \left(n \psi_{0}\right)>0\right), \\
\phi_{e}^{n-2} & =\frac{-1-2 \beta}{A} \quad\left(\cos \left(n \psi_{0}\right)<0\right),
\end{aligned}
$$

i.e.

$$
A \phi_{e}^{n-2}=\sigma-2 \beta
$$

where

$$
\sigma \equiv \operatorname{sign} \cos \left(n \psi_{0}\right)
$$

\footnotetext{
${ }^{11} \mathrm{At}$ the sample values

$$
v_{M}=10^{-3} m_{\mathrm{Pl}}, \beta=0.05, \Lambda=m_{\mathrm{Pl}}, \cos \left(n \psi_{0}\right)=0.5, \kappa_{\phi}=1,
$$

this approximation deviates from the exact form of $V$ by less than $10^{-4} \%$ in the range $\phi<0.1 v_{M}$ for $n=3$. For higher $n$ the approximation becomes even much better. We will therefore use the approximate form (3.38) of $V$ for the remainder of the paper.
} 


\subsection{Number of e-folds and observables}

The number of e-folds between the epoch of horizon-exit of the scale $k_{*}=0.002 \mathrm{Mpc}$ at inflaton field value $\phi_{*}$ and the end of inflation at inflaton field value $\phi_{e}$ in the slow-roll approximation is given by

$$
N_{*}=-\frac{1}{m_{\mathrm{Pl}}^{2}} \int_{\phi_{*}}^{\phi_{e}} \frac{V}{V^{\prime}} d \phi .
$$

Using again the approximate potential (3.38) and $V \approx M^{4}$ one obtains

$$
N_{*}=\left.\frac{1}{2 \beta(n-2)} \ln \frac{\phi^{n-2}}{2 \beta(n-1)+A \phi^{n-2}}\right|_{\phi_{*}} ^{\phi_{e}} .
$$

This implies the consistency condition

$$
2 \beta(n-1)+A \phi^{n-2}>0
$$

which, according to

$$
V^{\prime}\left(\phi, \psi_{0}\right) \approx-\frac{M^{4} \phi}{(n-1) m_{\mathrm{Pl}}^{2}}\left(2 \beta(n-1)+A \phi^{n-2}\right),
$$

physically means that $V^{\prime}<0$ during the whole of inflation. For positive $A$ (i.e. $\cos \left(n \psi_{0}\right)>$ 0 ) this is fulfilled for every value of $\phi$. For negative $A$ it implies the bound

$$
\phi^{n-2}<\frac{2 \beta(n-1)}{|A|}
$$

which, at the end of inflation, implies

$$
|A| \phi_{e}^{n-2}=1+2 \beta<2 \beta(n-1) \Rightarrow \beta>\frac{1}{2(n-2)} .
$$

Solving eq. (3.45) for $\phi_{*}$ gives

$$
A \phi_{*}^{n-2}=2(n-1) \beta \frac{1}{\frac{\sigma+(2 n-4) \beta}{\sigma-2 \beta} e^{2(n-2) \beta N_{*}}-1} .
$$

Note that for positive $A$, for every number of e-folds there is a solution as long as $\phi_{*}$ is close enough to zero. For negative $A$, there is an upper bound for $N_{*}$ given by the condition that the $\phi_{*}$ of eq. (3.50) must be positive. However, when in the following we will discuss the predictions for the spectral index, it will turn out that, for moderate $n, \beta$ must be very close to zero, which means that the condition (3.49) cannot be satisfied, and $\cos \left(n \psi_{0}\right)$ and thus also $A$ must be positive. We will therefore set $\sigma=+1$ for the remainder of the paper.

The expressions

$$
\begin{aligned}
n_{S} & \approx 1-6 \epsilon_{*}+2 \eta_{*}, \\
r & \approx 16 \epsilon_{*}, \\
\left.\frac{d n_{S}}{d \ln k}\right|_{k_{*}} & \approx-16 \epsilon_{*} \eta_{*}+24 \epsilon_{*}^{2}+2 \xi_{*}^{2}
\end{aligned}
$$



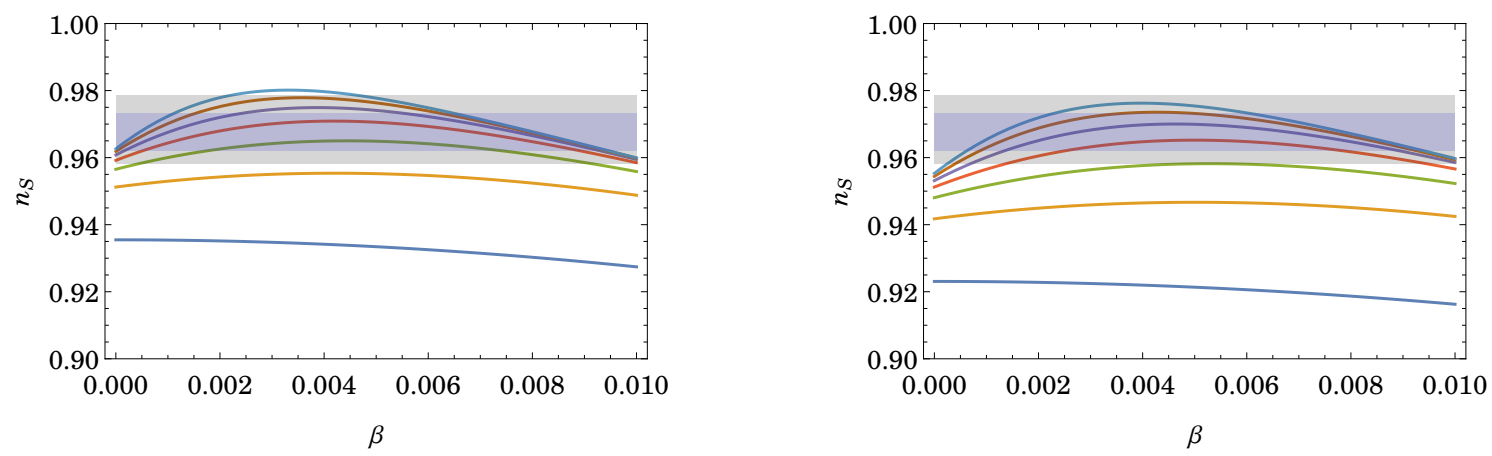

Figure 1. The spectral index of our model as a function of $\beta$ for different values of $n$ (lowest line: $n=3$, uppermost line: $n=9$ ). The purple and gray bands are the $68 \%$ and $95 \%$ C.L. intervals for $n_{S}$ as determined by the Planck Collaboration in [44] (Planck TT +lowP + BKP +lensing +ext). Left: $N_{*}=60$, Right: $N_{*}=50$.

for the CMB observables have to be evaluated at the field value $\phi_{*}$. In this way, we obtain a relation between the number of e-folds and $n_{S}, r$ and the running of the spectral index. As discussed earlier, $\epsilon$ effectively vanishes in our model, and the tensor to scalar ratio $r$ will be indistinguishable from zero. Therefore, the interesting predictions will be the ones for $n_{S}$ and its running. Since $\epsilon$ is negligibly small, we find

$$
n_{S}=1+2 \eta_{*}=1-4 \beta\left\{1+\frac{n-1}{\frac{1+(2 n-4) \beta}{1-2 \beta} e^{2(n-2) \beta N_{*}}-1}\right\} .
$$

For $\beta \rightarrow 0$ and using $N_{*} \gg \mathcal{O}(1)$ one obtains the approximate relation

$$
n_{S} \simeq 1-\frac{2(n-1)}{N_{*}(n-2)},
$$

which coincides with the result of [21]. Figure 1 shows the spectral index as a function of $\beta$ for different values of $N_{*}$. The running of the spectral index, due to the smallness of $\epsilon$, is given by

$$
\left.\frac{d n_{S}}{d \ln k}\right|_{k_{*}} \approx 2 \xi_{*}^{2}
$$

Using the approximate potential of eq. (3.38) and $V \approx M^{4}$ in the denominator of the definition (3.30c), and keeping only the lowest order term in $\phi / v_{M}$ and $\phi / m_{\mathrm{Pl}}$ one finds

$$
\xi \approx 2 \beta(n-2) A \phi^{n-2}
$$

i.e.

$$
\xi_{*} \approx \frac{4 \beta^{2}(n-1)(n-2)}{\frac{1+(2 n-4) \beta}{1-2 \beta} e^{2(n-2) \beta N_{*}}-1},
$$

where we have, as discussed for $\eta_{*}$, set $\sigma=+1$. A numerical evaluation of $\xi_{*}$ shows that for $\beta \in[0,0.01], n=3, \ldots, 9$ and $N_{*}=60$ or $N_{*}=50$ one has

$$
\left.\frac{d n_{S}}{d \ln k}\right|_{k_{*}}<10^{-6}
$$




\subsection{Examples: $n=6$ and $n=9$}

We will finally investigate two examples, $n=6$ (i.e. a $\mathbb{Z}_{12}$-symmetry in the scalar and superpotential) and $n=9$ (i.e. a $\mathbb{Z}_{9}$-symmetry in the scalar and superpotential), in the light of all the derived constraints.

We will start by asking the question whether the right-handed neutrinos $N_{R}$ produced via inflaton decay can be thermal. This will determine whether any subsequent leptogenesis is thermal or non-thermal. In order for this to be the case, we need

$$
T_{R}>m_{N}
$$

where

$$
m_{N}=\frac{1}{\sqrt{2}} \lambda v_{M}
$$

is the right-handed neutrino mass. From eqs. (3.16) and (3.18) one can derive the condition

$$
\frac{\Lambda^{2 n-4}}{v_{M}^{2 n-6} m_{\mathrm{Pl}}^{2}}<\frac{45 n^{2}}{2^{n+4} \pi^{4} g_{*}}
$$

for thermal right-handed neutrinos. For $n=6$ this gives

$$
\frac{\Lambda^{8}}{v_{M}^{6} m_{\mathrm{Pl}}^{2}}<\frac{405}{256 \pi^{4} g_{*}} \approx 7 \times 10^{-5}
$$

where we have used $g_{*}=240$ for the MSSM including three right-handed (s)neutrinos. This is a very strong constraint, and it will usually not be fulfilled, unless the flavour symmetry breaking scale $v_{M}$ and the cutoff scale $\Lambda$ are very close to each other. This means that typically at least the right-handed neutrino coupling to the inflaton will be non-thermally produced. Consequently, $g_{*}<240$, but we assume that all MSSM particles apart from one (or more) right-handed (s)neutrinos are produced thermally and thus use the approximation $g_{*} \approx 240$ in the following. ${ }^{12}$ The values of $\Lambda$ and $v_{M}$ for which, for $n=6$, the right-handed neutrinos produced by inflaton decay thermalize are shown in the upper left part of figure 2 .

Since we do, therefore, not impose a thermal $N_{R}$, the main condition for reheating to be possible is the kinematical requirement

$$
m_{\mathrm{Inf}}>2 m_{N}
$$

which is easily satisfied by an appropriate choice of $\lambda$. The other constraint on the success of our model is the condition $\Delta V \gg T_{R}^{4}$ for the successful avoidance of domain walls.

For these considerations only three physical parameters are relevant, the cutoff scale $\Lambda$, the mass scale $M$ and the Yukawa coupling $\lambda$ of the superpotential. Fixing $\Lambda$ to a given value, the other two quantities may be expressed in terms of the flavour symmetry breaking scale (inflaton=Majoron vev) $v_{M}=\sqrt{2}\left(M^{2} \Lambda^{n-2}\right)^{1 / n}$ and the right-handed neutrino mass

\footnotetext{
${ }^{12}$ The precise numerical value of $g_{*}$ does not have any influence on the qualitative features of our model we discuss in this section - see eqs. (3.61) and (3.67). Therefore, all results obtained here are also valid for reheat temperatures of the order of the top mass or smaller.
} 
$m_{N}=\lambda v_{M} / \sqrt{2}$. We will therefore show the allowed parameter regions of our model, for fixed $\Lambda$, in a plot with the values of $v_{M}$ and $m_{N}$ shown on the axes. Since the inflaton mass

$$
m_{\mathrm{Inf}}=n M\left(\frac{M}{\Lambda}\right)^{1-\frac{2}{n}}=\frac{n}{\sqrt{2}} v_{M}\left(\frac{v_{M}}{\sqrt{2} \Lambda}\right)^{n-2}=[n=6]=\frac{3}{2 \sqrt{2}} \frac{v_{M}^{5}}{\Lambda^{4}}
$$

is a function of $v_{M}$ and $\Lambda$ only, we may replace the $v_{M}$-axis by an $m_{\text {Inf-axis. }}$ -

The condition $m_{\text {Inf }}>2 m_{N}$ leads to

$$
m_{N}<\frac{n}{2 \sqrt{2}} v_{M}\left(\frac{v_{M}}{\sqrt{2} \Lambda}\right)^{n-2}=[n=6]=\frac{3}{4 \sqrt{2}} \frac{v_{M}^{5}}{\Lambda^{4}},
$$

i.e.

$$
\lambda<\frac{n}{2}\left(\frac{v_{M}}{\sqrt{2} \Lambda}\right)^{n-2}=[n=6]=\frac{3}{4}\left(\frac{v_{M}}{\Lambda}\right)^{4} .
$$

In order to find the constraints on the parameter space with respect to the condition $\Delta V \ll T_{R}^{4}$, we express the two quantities in terms of $m_{N}, v_{M}$ and $\Lambda$ :

$$
\begin{aligned}
\Delta V & =\Lambda^{4}\left(\frac{v_{M}}{\sqrt{2} \Lambda}\right)^{2 n}\left(4-\frac{\sqrt{6} n}{2 \pi} \frac{m_{\mathrm{Pl}} m_{N}^{2}}{v_{M}^{3}}\right), \\
T_{R}^{4} & =\Lambda^{4}\left(\frac{v_{M}}{\sqrt{2} \Lambda}\right)^{2 n} \frac{45 n^{2}}{16 \pi^{4} g_{*}} \frac{m_{\mathrm{Pl}}^{2} m_{N}^{4}}{v_{M}^{6}} .
\end{aligned}
$$

The border to the region excluded by the domain wall problem is then given by $\Delta V / T_{R}^{4}=1$ which can be expressed as

$$
m_{N}^{2}=\frac{4 \sqrt{6} \pi^{2}}{45 n} \frac{v_{M}^{3}}{m_{\mathrm{Pl}}}\left(-g_{*} \pi+\sqrt{g_{*}^{2} \pi^{2}+30 g_{*}}\right) .
$$

For $n=6$ and $g_{*}=240$ this gives the bound

$$
m_{N}<1.31 \frac{v_{M}^{3 / 2}}{m_{\mathrm{Pl}}^{1 / 2}}
$$

The upper right panel of figure 2 shows the allowed parameter space for $n=6$ and $\Lambda=m_{\mathrm{Pl}}$. An evident feature is the rather low reheat temperature for $v_{M} \lesssim M_{\mathrm{GUT}}$. Therefore, if at least all SM particles should be thermalized at the end of reheating, i.e. $T_{R} \gtrsim 200 \mathrm{GeV}$, we must have $v_{M} \gtrsim 10^{16} \mathrm{GeV}$. The lower half of figure 2 shows the same plots for $\Lambda=0.1 m_{\mathrm{Pl}}$ and $\Lambda=0.01 m_{\mathrm{Pl}}$. For these scenarios the inflaton mass will be much larger and, consequently, much higher right-handed neutrino masses $m_{N}$ are possible.

Finally, we also show the allowed parameter space for $n=9$ in figure 3. Qualitatively, figures 2 and 3 look similar with, however, the parameter space for $n=9$ being more constrained. 

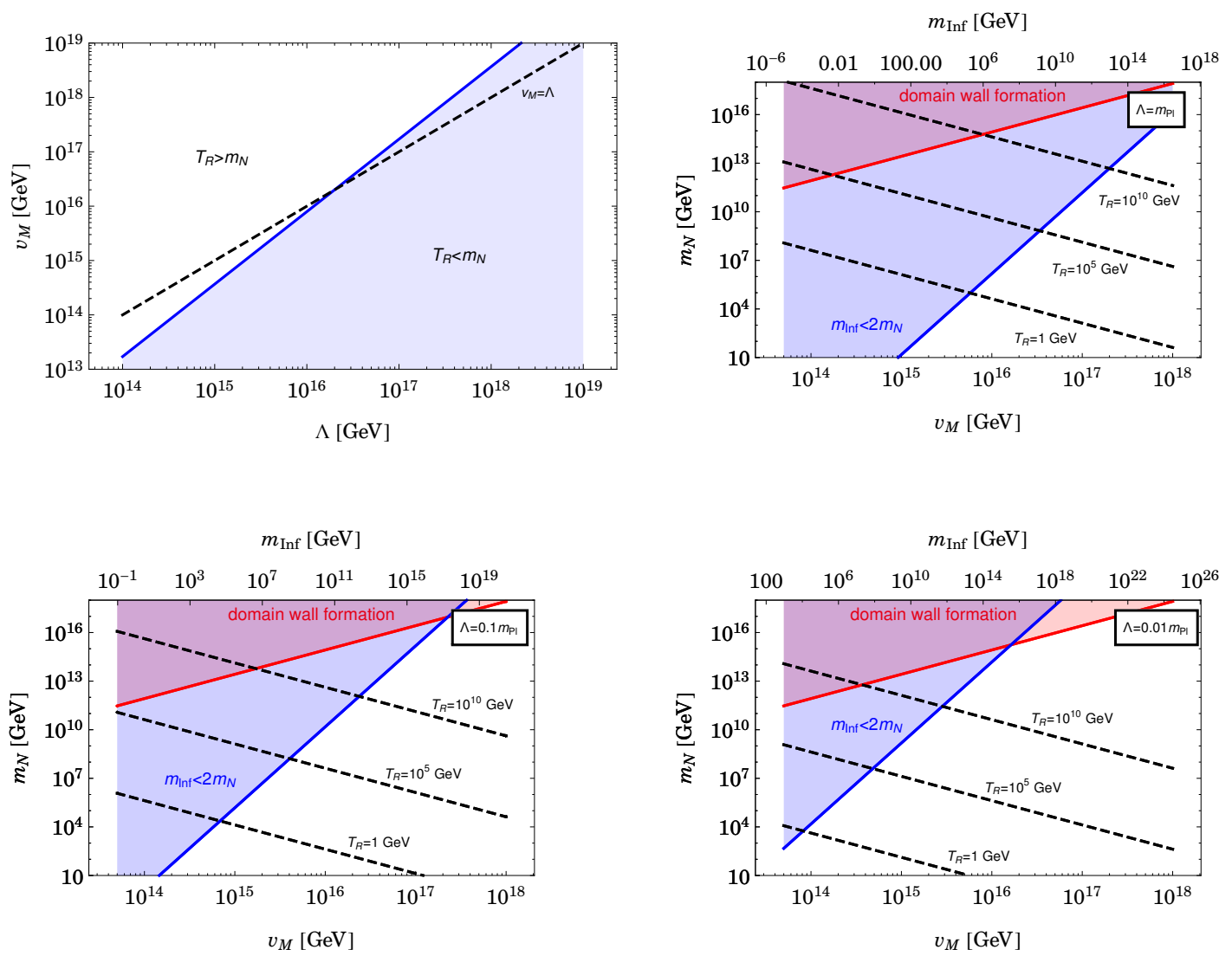

Figure 2. The allowed regions (in white) of the parameter space for $n=6$. Upper left: this plot shows the values of $v_{M}$ as a function of $\Lambda$ such that $T_{R}>m_{N}$, i.e. the right-handed neutrinos thermalize. The dashed black line indicates $v_{M}=\Lambda$. Therefore, if we impose the flavour symmetry breaking scale to be lower than the cutoff scale $\Lambda$, only the white area between the blue line and the dashed line for $\Lambda \lesssim 10^{16} \mathrm{GeV}$ is allowed. Upper right: the allowed parameter space (in white) for $n=6$ and $\Lambda=m_{\mathrm{Pl}}$. The blue shaded area is excluded due to the kinematic condition $m_{\mathrm{Inf}}>2 m_{N}$, and the red shaded area is excluded due to domain wall formation. The three dashed lines are the lines of constant reheat temperature $1 \mathrm{GeV}, 10^{5} \mathrm{GeV}$ and $10^{10} \mathrm{GeV}$, respectively. Lower left and lower right: the same plots for $\Lambda=0.1 m_{\mathrm{Pl}}$ and $\Lambda=0.01 m_{\mathrm{Pl}}$, respectively.

\subsection{Extension to more than one neutrino field}

Up to now we have considered only one neutrino field the inflaton couples to. However, the generalization to more than one neutrino is straight forward. Since reheating is the only process related to inflation where the right-handed neutrinos play a role, only the expression for $T_{R}$ will change. Namely, if the neutrino-inflaton coupling is extended to

$$
\mathcal{L}_{\Phi N N}=-\sum_{i} \lambda_{i} \Phi \overline{N_{i R}^{c}} N_{i R}+\text { H.c. }
$$

with neutrino mass eigenfields $N_{i}$, the decay width of the inflaton will generalize to

$$
\Gamma_{\phi}=\frac{m_{\operatorname{Inf}}}{16 \pi}\left|\sum_{i} \lambda_{i}\right|^{2} .
$$



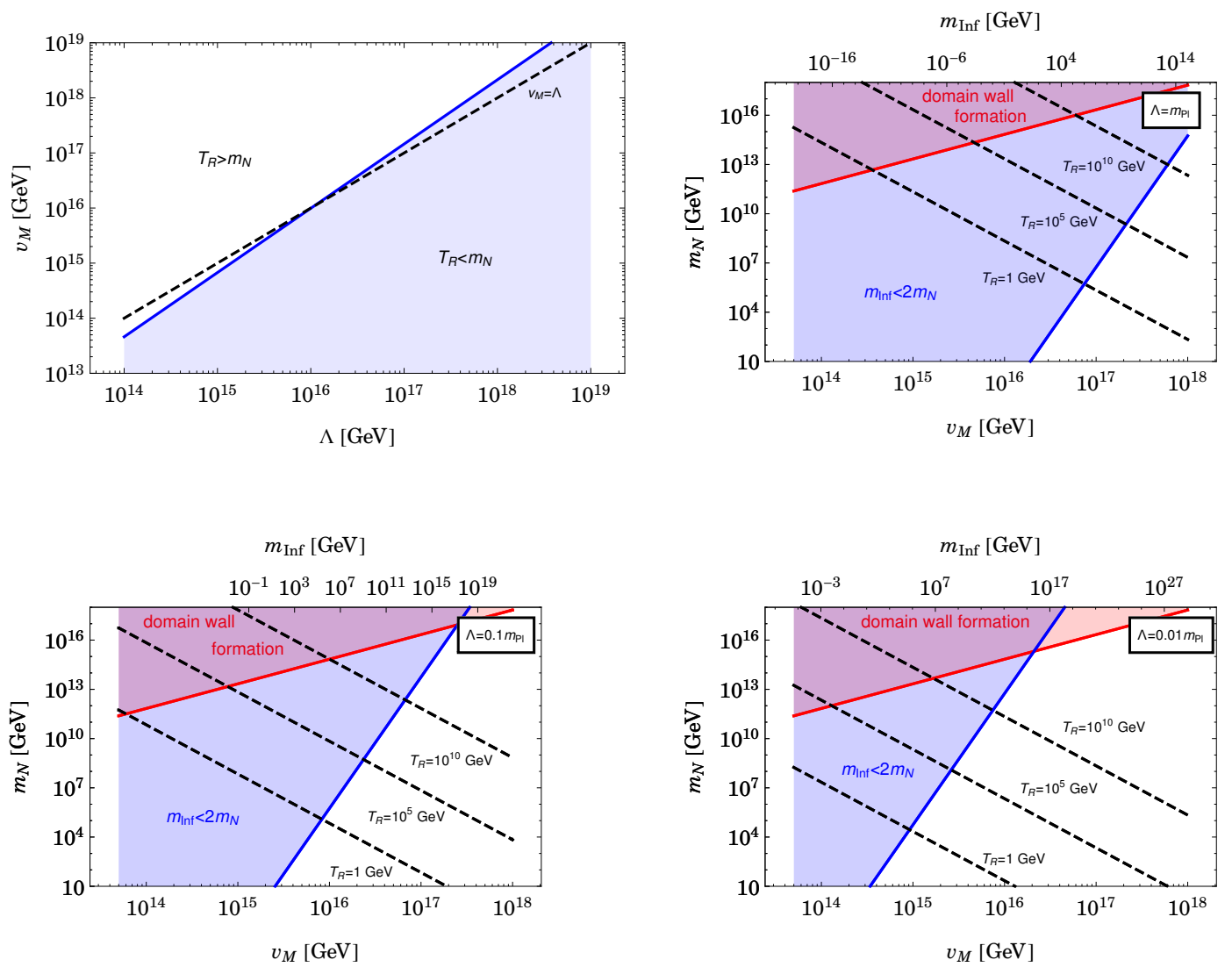

Figure 3. The allowed regions (in white) of the parameter space for $n=9$. Upper left: this plot shows the values of $v_{M}$ as a function of $\Lambda$ such that $T_{R}>m_{N}$, i.e. the right-handed neutrinos thermalize. The dashed black line indicates $v_{M}=\Lambda$. Upper right: the allowed parameter space (in white) for $n=9$ and $\Lambda=m_{\mathrm{Pl}}$. The blue shaded area is excluded due to the kinematic condition $m_{\text {Inf }}>2 m_{N}$, and the red shaded area is excluded due to domain wall formation. The three dashed lines are the lines of constant reheat temperature $1 \mathrm{GeV}, 10^{5} \mathrm{GeV}$ and $10^{10} \mathrm{GeV}$, respectively. Lower left and lower right: the same plots for $\Lambda=0.1 m_{\mathrm{Pl}}$ and $\Lambda=0.01 m_{\mathrm{Pl}}$, respectively.

Consequently, the coupling $\lambda$ in the expression for the reheat temperature has to be replaced by the effective coupling

$$
\tilde{\lambda} \equiv\left|\sum_{i} \lambda_{i}\right| .
$$

Since in the framework outlined in this paper all right-handed neutrinos would get their masses via the inflaton acquiring a vev, and since the right-handed neutrinos are usually expected to have strongly hierarchical masses (due to the seesaw relation), we will have

$$
\tilde{\lambda} \approx \max _{i}\left|\lambda_{i}\right|
$$

i.e. the reheat temperature will, to a very good approximation, be determined by the Yukawa-coupling of the heaviest right-handed neutrino. The figures 2 and 3 will therefore remain unchanged, if $m_{N}$ on the y-axis is the mass of the heaviest right-handed neutrino. 


\section{Conclusions}

In this paper we have considered a supersymmetric Majoron model in which the Majoron field $\Phi$ is responsible for both inflation and the generation of right-handed neutrino masses. In our model, global lepton number $\mathrm{U}(1)_{L}$ is explicity broken to $\mathbb{Z}_{N}$ by high powers of the Majoron field $\Phi^{n}$ in the superpotential and potential. Such terms explicitly break global $\mathrm{U}(1)_{L}$ down to a discrete subgroup of lepton number $\mathbb{Z}_{N}$ which is subsequently spontaneously broken by the vacuum expectation value of the Majoron, $v_{M}=\langle\Phi\rangle$. We have focussed on the possibility that, before spontaneous symmetry breaking, the scalar potential, including non-minimal Kähler corrections, is suitable for new or "hilltop" inflation. This is interesting since it relates inflation to the mechanism responsible for the origin of neutrino masses.

The usual motivation for supersymmetry in the context of inflation models, is that inflation depends on scalar degrees of freedom, and the dynamics of inflation is sensitive to high energy scales. This motivates supersymmetry and supergravity since such theories generally allow better control over the high energy dynamics of scalars. Although, due to supersymmetry, $\Phi$ necessarily is a complex scalar, i.e. with two real components, we have shown that, during inflation, the ratio of imaginary and real part $\operatorname{Im} \Phi / \operatorname{Re} \Phi$ evolves slowly compared to the expansion rate of the Universe, so the model may be treated as a single field inflation model. We have discussed domain wall creation due to thermal fluctuations due to the reheating process, and shown that, barring quantum fluctuations, after inflation the entire observable Universe would be expected to settle into a single global minimum. We have computed the spectral index $n_{S}$ which depends on only one free parameter (from the Kähler potential), which can be tuned to be compatible with the observed Planck value. This agrees with the results of [21] which uses a very similar superpotential (the only effective difference with respect to the predictions for the CMB observables being the restriction to even values of $n$ ). The degree of fine-tuning required in the Kähler potential is moderate. The only parameters affecting the Planck observables are $\beta=\left(\kappa_{X_{0} \Phi}-1\right) / 2$, which needs to be $<10^{-2}$ and $\kappa_{\Phi}$ which we have assumed to be small enough to justify the approximation of canonically normalized kinetic terms. These tunings are at a similar level to those required for the electroweak Higgs sector of supersymmetry. We have shown that tensor modes are small as expected from "hilltop" inflation, $r \approx 0$, and also the running of $n_{S}$ is negligible.

We investigated numerically two examples, $n=6$ corresponding to a discrete lepton number $\mathbb{Z}_{12}$ and $n=9$ corresponding to $\mathbb{Z}_{9}$. For both examples, it turns out that the righthanded neutrino mass is larger than the reheat temperature $T_{R}$ for values of the cut-off scale $\Lambda$ above the GUT scale. The inflaton may decay into pairs of right-handed neutrinos providing its mass is large enough, $m_{\operatorname{Inf}}>2 m_{N}$, which may be achieved if $v_{M}=\langle\Phi\rangle$ is large enough (but not exceeding the cut-off $\Lambda$ ). For both examples, we have shown that this may be achieved for a range of right-handed neutrino masses, $m_{N} \sim 10^{1}-10^{16} \mathrm{GeV}$, where the lower bound on $m_{N}$ comes from requiring that $T_{R} \gtrsim 1 \mathrm{GeV}$ and $v_{M}<\Lambda$, and the upper bound on $m_{N}$ comes from requiring that domain walls are not formed at the end of inflation. ${ }^{13}$ We also considered extending the model to the case of three right-handed

\footnotetext{
${ }^{13}$ If one would like to extend the present model to incorporate also leptogenesis, the reheat temperature must be at least $\mathcal{O}(100) \mathrm{GeV}$ in order to allow the sphaleron process. In this case the lower bound for $m_{N}$ will become $m_{N} \gtrsim 10^{3}-10^{4} \mathrm{GeV}$ instead of $m_{N} \gtrsim 10 \mathrm{GeV}$.
} 
neutrinos and argued that, if they have hierarchical masses, then the above results will apply to the heaviest right-handed neutrino.

We note that in our model the mass of the physical scalar $\phi$ and the pseudoscalar $\psi$ components of the complex Majoron field $\Phi$ are both equal to $m_{\text {Inf }}$ at the global minimum of the potential. Thus, there are two degenerate physical particles with common mass $m_{\phi}=m_{\psi}=m_{\text {Inf }}$, which in principle may be observable in future collider experiments, if $m_{\text {Inf }}$ is low enough. However, for $T_{R} \gtrsim 1 \mathrm{GeV}$, we find $m_{\mathrm{Inf}} \gtrsim 10^{5} \mathrm{GeV}$, making them practically unobservable at present or planned future colliders.

Finally we mention that, since this is a new proposal, there are inevitably several aspects of model building and cosmology which are beyond the scope of this paper. In particular, we have not considered a complete flavour model from which such a Majoron inflation model could emerge. In such a realistic model, the Majoron field might carry additional flavour quantum numbers, and there might be other fields in such models which could also play a role in cosmology. We have also not considered the effects of reheating beyond our naive estimates, nor leptogenesis, which would most likely be non-thermal due to the fact that $T_{R}<m_{N}$ over most of the parameter space consistent with $v_{M}<\Lambda$. These are all interesting aspects which are worth studying in the future.

In conclusion, we find that supersymmetric Majoron inflation is a promising and new idea which relates inflation to neutrino masses via the type-I seesaw mechanism. Indeed we have shown that, within the framework of non-minimal supergravity, the Majoron field $\Phi$ responsible for generating right-handed neutrino masses may also be suitable for giving low scale "hilltop" inflation, with a discrete lepton number $\mathbb{Z}_{N}$ spontaneously broken at the end of inflation. The key features of this newly proposed framework are:

1. Though $\Phi$ is necessarily complex, we have shown that the model can be treated as a single-field inflation model involving $|\Phi|$.

2. The domain wall problem can be avoided in a very large part of the parameter space.

3. In most parts of the allowed parameter space the heaviest right-handed neutrino will be non-thermally produced and the reheat temperature will be small $\left(\lesssim 10^{10} \mathrm{GeV}\right)$ for inflaton vevs smaller than $M_{\mathrm{GUT}}$.

\section{Acknowledgments}

P.O.L. wants to thank Alexander Merle for helpful discussions. The authors acknowledge support from the STFC grant ST/L000296/1 and the European Union Horizon 2020 research and innovation programme under the Marie Sklodowska-Curie grant agreements InvisiblesPlus RISE No. 690575 and Elusives ITN No. 674896.

\section{A Treatment of the model as a single-field inflation model}

Since our inflation model is supersymmetric, $\Phi$ is necessarily complex, and we have to treat the model as a two-field inflation model, with the two real fields being $\phi_{R}=\sqrt{2} \operatorname{Re} \Phi$ 
and $\phi_{I}=\sqrt{2} \operatorname{Im} \Phi .{ }^{14}$ The purpose of this appendix is to show that, during the inflationary epoch, the ratio of these two fields is effectively frozen, with inflationary dynamics controlled by the magnitude of the complex Majoron field $|\Phi|$.

We rewrite $\Phi$ in terms of $\phi_{R}$ and $\phi_{I}$, i.e.

$$
\Phi \equiv \frac{1}{\sqrt{2}}\left(\phi_{R}+i \phi_{I}\right)
$$

which leads to

$$
\mathcal{L}=\frac{1}{2}\left(\partial_{\mu} \phi_{R}\right)\left(\partial^{\mu} \phi_{R}\right)+\frac{1}{2}\left(\partial_{\mu} \phi_{I}\right)\left(\partial^{\mu} \phi_{I}\right)-V\left(\phi_{R}, \phi_{I}\right)
$$

From the energy-momentum tensor one then finds the energy density and pressure of the scalar fields,

$$
\begin{aligned}
\rho & =\frac{1}{2} \dot{\phi}_{R}^{2}+\frac{1}{2} \dot{\phi}_{I}^{2}+V\left(\phi_{R}, \phi_{I}\right), \\
p & =\frac{1}{2} \dot{\phi}_{R}^{2}+\frac{1}{2} \dot{\phi}_{I}^{2}-V\left(\phi_{R}, \phi_{I}\right),
\end{aligned}
$$

and the equations of motion are given by

$$
\begin{aligned}
& \ddot{\phi}_{R}+3 H \dot{\phi}_{R}+\frac{\partial V}{\partial \phi_{R}}=0, \\
& \ddot{\phi}_{I}+3 H \dot{\phi}_{I}+\frac{\partial V}{\partial \phi_{I}}=0, \\
& H^{2}=\frac{1}{3 m_{\mathrm{Pl}}^{2}}\left(V+\frac{1}{2} \dot{\phi}_{R}^{2}+\frac{1}{2} \dot{\phi}_{I}^{2}\right) .
\end{aligned}
$$

In the slow-roll approximation $\ddot{\phi}_{R, I} \ll 3 H \dot{\phi}_{R, I}, \dot{\phi}_{R, I}^{2} \ll V$ and one finds

$$
\begin{aligned}
3 H \dot{\phi}_{R} & \approx-\frac{\partial V}{\partial \phi_{R}}, \\
3 H \dot{\phi}_{I} & \approx-\frac{\partial V}{\partial \phi_{I}}, \\
H^{2} & \approx \frac{V}{3 m_{\mathrm{Pl}}^{2}} .
\end{aligned}
$$

The value of the potential during the slow-roll phase is well approximated by its value at vanishing field, i.e. $V \approx V(0) \equiv V_{0}=M^{4}$ and thus

$$
H^{2} \approx \frac{V_{0}}{3 m_{\mathrm{Pl}}^{2}} .
$$

In the following we will show that during inflation the model can effectively be treated as a single-field inflation model. To do so, we study the time evolution of the ratio between the imaginary and real part of $\Phi$,

$$
\alpha \equiv \frac{\operatorname{Im} \Phi}{\operatorname{Re} \Phi}=\frac{\phi_{I}}{\phi_{R}}
$$

\footnotetext{
${ }^{14}$ The factors of $\sqrt{2}$ are introduced for convenience to obtain a Lagrangian in terms of real fields which is canonically normalized: $\mathcal{L}=\frac{1}{2}\left(\partial_{\mu} \phi_{R}\right)^{2}+\frac{1}{2}\left(\partial_{\mu} \phi_{I}\right)^{2}+\ldots$.
} 
during inflation. The equation of motion for $\alpha$ is found from eqs. (A.5a) and (A.5b):

$$
\frac{\dot{\alpha}}{\alpha}=\frac{\dot{\phi}_{I}}{\phi_{I}}-\frac{\dot{\phi}_{R}}{\phi_{R}}=-\frac{1}{3 H}\left(\frac{1}{\phi_{I}} \frac{\partial V}{\partial \phi_{I}}-\frac{1}{\phi_{R}} \frac{\partial V}{\partial \phi_{R}}\right) .
$$

In new inflation models the field value is always much smaller than $m_{\mathrm{Pl}}$, and we can, for the moment, safely set $m_{\mathrm{Pl}} \rightarrow \infty$. In this limit we find

$$
\frac{\dot{\alpha}}{\alpha}=\frac{n M^{2}}{3 \sqrt{2}^{n} \Lambda^{n-2} H} \phi_{R}^{n-2} \frac{i}{\alpha}\left((1+i \alpha)^{n}-(1-i \alpha)^{n}\right) .
$$

The solution of this differential equation is given by

$$
\int_{\alpha\left(t_{0}\right)}^{\alpha} \frac{d \alpha^{\prime}}{i\left(\left(1+i \alpha^{\prime}\right)^{n}-\left(1-i \alpha^{\prime}\right)^{n}\right)}=\frac{n M^{2}}{3 \sqrt{2}^{n}} \int_{t_{0}}^{t} d t^{\prime} \frac{1}{H\left(t^{\prime}\right)}\left(\frac{\phi_{R}\left(t^{\prime}\right)}{\Lambda}\right)^{n-2},
$$

where $t_{0}$ is the time when inflation starts and $\alpha\left(t_{0}\right)$ the ratio of imaginary to real part of $\Phi$ in the small patch of the pre-inflationary Universe which is inflated to the present (and in the future observable) Universe. The integrand of the integral on the left-hand side of eq. (A.10) is the inverse of a polynomial in $\alpha$, and the integral therefore has the form

$$
\int_{\alpha\left(t_{0}\right)}^{\alpha} \frac{d \alpha^{\prime}}{i\left(\left(1+i \alpha^{\prime}\right)^{n}-\left(1-i \alpha^{\prime}\right)^{n}\right)}=\ln \frac{F(\alpha)}{\left.F\left(\alpha\left(t_{0}\right)\right)\right)},
$$

where $F(\alpha)$ is a rational function of $\alpha$. The solution may therefore be recast as

$$
F(\alpha(t))=F\left(\alpha\left(t_{0}\right)\right) \times \exp \left(\frac{n M^{2}}{3 \sqrt{2}^{n}} \int_{t_{0}}^{t} d t^{\prime} \frac{1}{H\left(t^{\prime}\right)}\left(\frac{\phi_{R}\left(t^{\prime}\right)}{\Lambda}\right)^{n-2}\right) .
$$

Since the Hubble constant $H$ is, during inflation, also constant in time, we find

$$
\int_{t_{0}}^{t} d t^{\prime} \frac{1}{H\left(t^{\prime}\right)}\left(\frac{\phi_{R}\left(t^{\prime}\right)}{\Lambda}\right)^{n-2}=\frac{1}{\Lambda^{n-2} H\left(t_{0}\right)} \int_{t_{0}}^{t} d t^{\prime} \phi_{R}\left(t^{\prime}\right)^{n-2} \equiv \frac{t-t_{0}}{\Lambda^{n-2} H\left(t_{0}\right)}{\overline{\phi_{R}}}^{n-2}
$$

where we have defined an average field value $\overline{\phi_{R}}$ during inflation by

$$
{\overline{\phi_{R}}}^{n-2} \equiv \frac{1}{t-t_{0}} \int_{t_{0}}^{t} d t^{\prime} \phi_{R}\left(t^{\prime}\right)^{n-2} .
$$

In this way we find the following expression for the time evolution of $\alpha$ during inflation:

$$
F(\alpha(t))=F\left(\alpha\left(t_{0}\right)\right) \times \exp \left(\frac{n M^{2}}{3 \sqrt{2}^{n} \Lambda^{n-2} H}{\overline{\phi_{R}}}^{n-2}\left(t-t_{0}\right)\right) .
$$

Therefore, if

$$
\frac{n M^{2}\left|\overline{\phi_{R}}\right|^{n-2}}{3 \sqrt{2}^{n} \Lambda^{n-2} H}<H
$$

during inflation, the evolution of $\alpha$ is slow compared to the expansion time scale and we can treat our model as a single field inflation model by setting $\alpha(t) \approx \alpha\left(t_{0}\right)$ and making the replacement

$$
\phi_{I} \approx \alpha\left(t_{0}\right) \phi_{R} .
$$


Using $H^{2} \approx M^{4} / 3 m_{\mathrm{Pl}}^{2}$, the condition (A.16) becomes

$$
\left|\overline{\phi_{R}}\right|^{n-2}<\frac{v_{M}^{n}}{n m_{\mathrm{Pl}}^{2}},
$$

where

$$
v_{M}=\sqrt{2}\left(M^{2} \Lambda^{n-2}\right)^{1 / n} .
$$

However, also the converse situation

$$
\left|\bar{\phi}_{R}\right|^{n-2}>\frac{v_{M}^{n}}{n m_{\mathrm{Pl}}^{2}}
$$

leads to an effective single-field inflation model. Namely, in this case, during the first few e-folds of inflation $\alpha$ rapidly approaches one of its asymptotic values (depending on the evolution of $\left.\overline{\phi_{R}}(t)\right)$

$$
\alpha_{\infty}=\tan (2 \pi k / n) \quad(k \in\{0, \ldots, n-1\}) .
$$

This corresponds to the $n$ equivalent minima of the $\mathbb{Z}_{n}$-symmetric potential, which in the complex plane lie in the directions of the real axis and the directions defined by the nontrivial $n$th roots of unity $\exp (2 \pi i k / n)$. Hence, in this scenario, the field can be assumed to be in one of its minima (with respect to $\alpha$ ) during inflation which means

$$
\phi_{I} \approx \alpha_{\infty} \phi_{R}
$$

again allowing to treat the model as a model of single-field inflation.

The equations of motion for the effective single-field inflation model are derived in appendix B. The result is a set of equations of motion for $\phi=\sqrt{\phi_{R}^{2}+\phi_{I}^{2}}$ which reads

$$
\begin{aligned}
& \ddot{\phi}+3 H \dot{\phi}+\frac{\partial V(\phi, \psi)}{\partial \phi}=0, \\
& H^{2}=\frac{1}{3 m_{\mathrm{Pl}}^{2}}\left(V+\frac{1}{2} \dot{\phi}^{2}\right),
\end{aligned}
$$

where $\psi=\arctan \alpha$ and the derivative has to be evaluated at the approximately constant value $\psi_{0}$ during inflation.

\section{B Derivation of the equation of motion in the effective single-field infla- tion framework}

The equations of motion for the two fields $\phi_{R}$ and $\phi_{I}$ defined as

are

$$
\Phi \equiv \frac{1}{\sqrt{2}}\left(\phi_{R}+i \phi_{I}\right) \equiv \frac{1}{\sqrt{2}} \phi e^{i \psi}
$$

$$
\begin{aligned}
& \ddot{\phi}_{R}+3 H \dot{\phi}_{R}+\frac{\partial V}{\partial \phi_{R}}=0, \\
& \ddot{\phi}_{I}+3 H \dot{\phi}_{I}+\frac{\partial V}{\partial \phi_{I}}=0, \\
& H^{2}=\frac{1}{3 m_{\mathrm{Pl}}^{2}}\left(V+\frac{1}{2} \dot{\phi}_{R}^{2}+\frac{1}{2} \dot{\phi}_{I}^{2}\right),
\end{aligned}
$$


i.e. eqs. (A.4). The equations of motion for the effective single-field inflation model can be derived by rewriting eqs. (B.2) in terms of the polar coordinates $\phi$ and $\psi$ :

$$
\begin{aligned}
\phi_{R} & =\phi \cos \psi, & \phi_{I} & =\phi \sin \psi, \\
\frac{\partial}{\partial \phi_{R}} & =\cos \psi \frac{\partial}{\partial \phi}-\sin \psi \frac{1}{\phi} \frac{\partial}{\partial \psi}, & \frac{\partial}{\partial \phi_{I}} & =\sin \psi \frac{\partial}{\partial \phi}+\cos \psi \frac{1}{\phi} \frac{\partial}{\partial \psi} .
\end{aligned}
$$

Adding $\cos \psi$ times eq. (B.2a) to $\sin \psi$ times eq. (B.2b) then reveals

$$
\ddot{\phi}+3 H \dot{\phi}+\frac{\partial V(\phi, \psi)}{\partial \phi}-\phi \dot{\psi}^{2}=0
$$

and similarly one obtains

$$
H^{2}=\frac{1}{3 m_{\mathrm{Pl}}^{2}}\left(V+\frac{1}{2} \dot{\phi}^{2}+\frac{1}{2} \phi^{2} \dot{\psi}^{2}\right)
$$

In the case where $\alpha=\phi_{I} / \phi_{R}$ evolves slowly compared to the expansion rate, or is already close to its asymptotic value - see the discussion in appendix $\mathrm{A}$ - we have $\dot{\alpha} \approx 0$ and hence $\dot{\psi} \approx 0$ and the equations of motion reduce to the following set of equations of motion for a single real scalar field $\phi=\sqrt{2}|\Phi|$ :

$$
\begin{aligned}
& \ddot{\phi}+3 H \dot{\phi}+\frac{\partial V}{\partial \phi}=0, \\
& H^{2}=\frac{1}{3 m_{\mathrm{Pl}}^{2}}\left(V+\frac{1}{2} \dot{\phi}^{2}\right) .
\end{aligned}
$$

This is exactly the form expected for a single-field inflation model. The derivative $\partial V / \partial \phi$ is to be taken at $\psi=\psi_{0}$, where $\psi_{0}$ is the (approximately constant) value of $\psi$ during inflation.

Open Access. This article is distributed under the terms of the Creative Commons Attribution License (CC-BY 4.0), which permits any use, distribution and reproduction in any medium, provided the original author(s) and source are credited.

\section{References}

[1] T. Ohlsson ed., Special issue on "Neutrino oscillations: celebrating the Nobel prize in physics 2015", Nucl. Phys. B 908 (2016) 1.

[2] G.B. Gelmini and M. Roncadelli, Left-handed neutrino mass scale and spontaneously broken lepton number, Phys. Lett. 99B (1981) 411 [INSPIRE].

[3] S. Weinberg, Baryon and lepton nonconserving processes, Phys. Rev. Lett. 43 (1979) 1566 [INSPIRE].

[4] P. Minkowski, $\mu \rightarrow$ e $\gamma$ at a rate of one out of $10^{9}$ muon decays?, Phys. Lett. B 67 (1977) 421 [INSPIRE].

[5] T. Yanagida, Horizontal symmetry and masses of neutrinos, in Proc. of the Workshop on Unified Theory and Baryon Number of the Universe, KEK, Japan, (1979) [INSPIRE].

[6] P. Ramond, The family group in grand unified theories, in Sanibel Talk, CALT-68-709, February 1979 [hep-ph/9809459] [INSPIRE]. 
[7] M. Gell-Mann, P. Ramond and R. Slansky, Complex spinors and unified theories, in Supergravity, North Holland, Amsterdam The Netherlands, (1979) [Conf. Proc. C 790927 (1979) 315] [arXiv: 1306.4669] [INSPIRE].

[8] S.L. Glashow, The future of elementary particle physics, in Cargese Lectures, Cargese France, (1979) [NATO Sci. Ser. B 61 (1980) 687] [InSPIRE].

[9] R.N. Mohapatra and G. Senjanović, Neutrino mass and spontaneous parity violation, Phys. Rev. Lett. 44 (1980) 912 [INSPIRE].

[10] Y. Chikashige, R.N. Mohapatra and R.D. Peccei, Are there real Goldstone bosons associated with broken lepton number?, Phys. Lett. B 98 (1981) 265 [INSPIRE].

[11] G.F. Giudice, A. Masiero, M. Pietroni and A. Riotto, The supersymmetric singlet Majoron, Nucl. Phys. B 396 (1993) 243 [hep-ph/9209296] [InSPIRE].

[12] M. Shiraishi, I. Umemura and K. Yamamoto, Spontaneous R-parity breaking in a supersymmetric Majoron model, Phys. Lett. B 313 (1993) 89 [InSPIRE].

[13] J.R. Espinosa, The supersymmetric singlet Majoron model and the general upper bound on the lightest Higgs boson mass, Phys. Lett. B 353 (1995) 243 [hep-ph/9503255] [INSPIRE].

[14] I. Umemura and K. Yamamoto, Neutrinos in the supersymmetric singlet Majoron model, Nucl. Phys. B 423 (1994) 405 [inSPIRE].

[15] R.N. Mohapatra and X. Zhang, Cosmological constraint on the scale of the supersymmetric singlet Majoron, Phys. Rev. D 49 (1994) 1163 [Erratum ibid. D 49 (1994) 6246] [hep-ph/9307231] [INSPIRE].

[16] A.H. Guth, The inflationary universe: a possible solution to the horizon and flatness problems, Phys. Rev. D 23 (1981) 347 [InSPIRE].

[17] D.H. Lyth and A. Riotto, Particle physics models of inflation and the cosmological density perturbation, Phys. Rept. 314 (1999) 1 [hep-ph/9807278] [INSPIRE].

[18] S.M. Boucenna, S. Morisi, Q. Shafi and J.W.F. Valle, Inflation and Majoron dark matter in the seesaw mechanism, Phys. Rev. D 90 (2014) 055023 [arXiv: 1404.3198] [InSPIRE].

[19] G. Ballesteros, J. Redondo, A. Ringwald and C. Tamarit, Unifying inflation with the axion, dark matter, baryogenesis and the seesaw mechanism, Phys. Rev. Lett. 118 (2017) 071802 [arXiv: 1608.05414] [INSPIRE].

[20] G. Ballesteros, J. Redondo, A. Ringwald and C. Tamarit, Standard Model-axion-seesaw-Higgs portal inflation. Five problems of particle physics and cosmology solved in one stroke, arXiv:1610.01639 [INSPIRE].

[21] V.N. Senoguz and Q. Shafi, New inflation, preinflation and leptogenesis, Phys. Lett. B 596 (2004) 8 [hep-ph/0403294] [INSPIRE].

[22] T. Asaka, K. Hamaguchi, M. Kawasaki and T. Yanagida, Leptogenesis in inflationary universe, Phys. Rev. D 61 (2000) 083512 [hep-ph/9907559] [INSPIRE].

[23] L. Boubekeur and D. Lyth, Hilltop inflation, JCAP 07 (2005) 010 [hep-ph/0502047] [INSPIRE].

[24] K. Kohri, C.-M. Lin and D.H. Lyth, More hilltop inflation models, JCAP 12 (2007) 004 [arXiv:0707.3826] [INSPIRE].

[25] K. Nakayama and F. Takahashi, PeV-scale supersymmetry from new inflation, JCAP 05 (2012) 035 [arXiv:1203.0323] [INSPIRE]. 
[26] S. Antusch and F. Cefalà, SUGRA new inflation with Heisenberg symmetry, JCAP 10 (2013) 055 [arXiv: 1306.6825] [INSPIRE].

[27] S. Antusch, D. Nolde and S. Orani, Hill crossing during preheating after hilltop inflation, JCAP 06 (2015) 009 [arXiv: 1503.06075] [INSPIRE].

[28] S. Antusch and S. Orani, Impact of other scalar fields on oscillons after hilltop inflation, JCAP 03 (2016) 026 [arXiv: 1511.02336] [INSPIRE].

[29] S.F. King, A to Z of flavour with Pati-Salam, JHEP 08 (2014) 130 [arXiv:1406.7005] [INSPIRE].

[30] S. Antusch, S.F. King, M. Malinsky, L. Velasco-Sevilla and I. Zavala, Flavon inflation, Phys. Lett. B 666 (2008) 176 [arXiv:0805.0325] [INSPIRE].

[31] S. Antusch and D. Nolde, Matter inflation with $A_{4}$ flavour symmetry breaking, JCAP 10 (2013) 028 [arXiv: 1306.3501] [INSPIRE].

[32] S. Antusch and D. Nolde, Realising effective theories of tribrid inflation: are there effects from messenger fields?, JCAP 09 (2015) 055 [arXiv: 1505.06910] [INSPIRE].

[33] S. Antusch, M. Bastero-Gil, S.F. King and Q. Shafi, Sneutrino hybrid inflation in supergravity, Phys. Rev. D 71 (2005) 083519 [hep-ph/0411298] [INSPIRE].

[34] H. Murayama, H. Suzuki, T. Yanagida and J. Yokoyama, Chaotic inflation and baryogenesis by right-handed sneutrinos, Phys. Rev. Lett. 70 (1993) 1912 [INSPIRE].

[35] J.R. Ellis, M. Raidal and T. Yanagida, Sneutrino inflation in the light of WMAP: reheating, leptogenesis and flavor violating lepton decays, Phys. Lett. B 581 (2004) 9 [hep-ph/0303242] [INSPIRE].

[36] J.R. Ellis, Sneutrino inflation, Nucl. Phys. Proc. Suppl. B 137 (2004) 190 [hep-ph/0403247] [INSPIRE].

[37] F. Björkeroth, S.F. King, K. Schmitz and T.T. Yanagida, Leptogenesis after chaotic sneutrino inflation and the supersymmetry breaking scale, Nucl. Phys. B 916 (2017) 688 [arXiv: 1608.04911] [INSPIRE].

[38] K. Nakayama, F. Takahashi and T.T. Yanagida, Viable chaotic inflation as a source of neutrino masses and leptogenesis, Phys. Lett. B 757 (2016) 32 [arXiv:1601.00192] [INSPIRE].

[39] S. Antusch, J.P. Baumann, V.F. Domcke and P.M. Kostka, Sneutrino hybrid inflation and nonthermal leptogenesis, JCAP 10 (2010) 006 [arXiv: 1007.0708] [INSPIRE].

[40] S. Khalil and A. Sil, Right-handed sneutrino inflation in SUSY B-L with inverse seesaw, Phys. Rev. D 84 (2011) 103511 [arXiv:1108.1973] [INSPIRE].

[41] S.F. King and Q. Shafi, Minimal supersymmetric $\mathrm{SU}(4) \times \mathrm{SU}(2)_{L} \times \mathrm{SU}(2)_{R}$, Phys. Lett. B 422 (1998) 135 [hep-ph/9711288] [INSPIRE].

[42] Ya. B. Zeldovich, I. Yu. Kobzarev and L.B. Okun, Cosmological consequences of the spontaneous breakdown of discrete symmetry, Zh. Eksp. Teor. Fiz. 67 (1974) 3 [Sov. Phys. JETP 40 (1974) 1] [INSPIRE].

[43] E.W. Kolb and M.S. Turner, The early universe, Front. Phys. 69 (1990) 1 [INSPIRE].

[44] Planck collaboration, P.A.R. Ade et al., Planck 2015 results. XIII. Cosmological parameters, Astron. Astrophys. 594 (2016) A13 [arXiv:1502.01589] [INSPIRE]. 